\section{La Révolution française}

Cahiers de l'Institut d'histoire de la Révolution française

$17 \mid 2020$

Gouverner par la loi

\title{
La gestion routière de la Haute-Garonne entre
}

1790-1796

\section{Daebo Kim}

\section{(2) OpenEdition}

1 Journals

Édition électronique

URL : http://journals.openedition.org/lrf/3656

DOI : $10.4000 /$ Irf.3656

ISSN : 2105-2557

\section{Éditeur}

IHMC - Institut d'histoire moderne et contemporaine (UMR 8066)

Référence électronique

Daebo Kim, «La gestion routière de la Haute-Garonne entre 1790-1796 », La Révolution française [En ligne], 17 | 2020, mis en ligne le 24 février 2020, consulté le 20 avril 2020. URL : http:// journals.openedition.org//rf/3656 ; DOI : https://doi.org/10.4000/lrf.3656

Ce document a été généré automatiquement le 20 avril 2020.

(c) La Révolution française 


\title{
La gestion routière de la Haute- Garonne entre 1790-1796
}

\author{
Daebo Kim
}

\section{NOTE DE L'ÉDITEUR}

Thèse soutenue par Daebo Kim et dirigée par M. le Professeur Pierre Serna, le 19 décembre 2017. Le jury était composé de Mesdames Anne Conchon (présidente) et Dominique Godineau (rapporteuse), et Monsieur Philippe Bourdin (rapporteur).

1 Cette thèse a pour but de repenser les ponts et chaussées durant la Révolution française sous l'angle d'une administration départementale. Dans l'histoire des ponts et chaussées ou des travaux publics, la Révolution française est généralement considérée comme une époque stérile en travaux publics par rapport à l'Ancien Régime et l'époque napoléonienne, parce que l'on résume souvent qu'il n'y eut pas de grands travaux entrepris pendant la décennie révolutionnaire. Alors, pourquoi les routes pendant la Révolution française doivent être recherchées? Autrement dit, quel mérite y aurait-il à initier une étude sur les routes et, de plus, sur celles au niveau d'un département nouveau-né de la Révolution française ? D'abord, l'étude sur l'administration des ponts et chaussées à tous les niveaux, parisiens et locaux, et sur l'exécution réelle des travaux nous permet d'aborder la question du degré de centralisation ou de décentralisation de cette institution pendant la Révolution française sous différents angles. De plus, la question de la centralisation implique immédiatement celle de la relation entre le pouvoir parisien et le pouvoir local.

Entre 1790 et 1796, même si le bilan économique de la période n'est pas entièrement catastrophique, le défaut de moyens financiers que la France éprouve rend de plus en 
plus difficile l'exécution normale des travaux routiers. Pourtant, cela ne signifie pas qu'il n'y a aucun effort de la part de l'administration pour les travaux des routes, lesquels prennent même une place prédominante dans l'administration départementale. Dans ce contexte, l'objet de cette étude est de comprendre comment se noue et évolue la relation entre le département de la Haute-Garonne et le pouvoir parisien dans le nouvel ordre politique et administratif installé par la Révolution française. Ensuite, dans le même fil, il s'agit de mettre en évidence le réseau formé par les différents acteurs des travaux routiers, notamment les entrepreneurs et le directoire du département, qui essaie d'élargir son contrôle dans son ressort pour reprendre et exécuter les travaux des routes. Alors que la Révolution s'affirme comme une nouvelle étape dans la tendance à la centralisation et à l'uniformisation, la compétence du pouvoir local en matière de travaux publics subit un changement important.

3 L'évolution de la relation entre pouvoir central et local se fait en trois temps. Dans un premier temps, de la fin de l'Ancien Régime jusqu'au moment où, en 1790, les commissions provisoires s'occupent d'administrer la fiscalité locale. Nous avons noté tant l'uniformisation et la centralisation opérée autour du Corps des Ponts et Chaussées que la décentralisation réalisée au sein des pays d'états, notamment dans le Languedoc, où le rôle centralisateur des états provinciaux a été relevé par Arlette Jouanna, qui évoque même une «bi-centralisation». Le développement du Corps des Ponts et Chaussées s'inscrit dans le contexte plus large du XviII siècle. L'établissement de ce Corps et l'ouverture de l'École des Ponts et Chaussées résultent de la volonté de diffuser le savoir technique par et pour les ingénieurs dans toute la France. Pour ce faire, la direction technique des ingénieurs répartis dans les généralités est renforcée. La centralisation se retrouve aussi quant à la répartition des fonds dont l'intendant des finances est responsable. Toutefois, il existe une limite importante à cette évolution : les pays d'états, ce particularisme d'Ancien Régime, et parmi eux la province de Languedoc, qui jouit de la plus grande autonomie. Dans ce pays méridional, l'institution des travaux publics est décentralisée à mesure que les états provinciaux éteignent progressivement l'influence de l'administration royale dans leur ressort. Profitant du vide administratif, ils affirment qu'ils sont le seul pouvoir qui détient le droit de financer les routes et de former le personnel technique, ce qui représente le plus haut niveau d'autonomie dans le domaine des travaux publics. Par conséquent, le contrôle du pouvoir royal sur les travaux routiers dans le Languedoc ne cesse de se relâcher jusqu'à la fin de l'Ancien Régime. Il en va ainsi jusqu'en 1790, année où les commissions provisoires héritent de la fiscalité, en attendant que la première administration locale de la Révolution française ne soit mise en place.

Ayant hérité du régime des ponts et chaussées de l'Ancien Régime et de ses nombreuses exceptions, La Révolution marque ensuite un tournant décisif dans la centralisation et l'uniformisation de l'administration des ponts et chaussées. À partir de 1791, quand le directoire du département entre véritablement en fonction, les Constituants optent résolument pour une administration uniforme et centralisée des ponts et chaussées. Ils déclarent qu'il n'y a qu'une administration centrale des ponts et chaussées et qu'une seule école des ponts et chaussées en France, et que toutes les institutions des travaux publics dans les pays d'états sont supprimées. L'administration centrale, composée du ministre de l'Intérieur et de l'Assemblée des Ponts et Chaussées, a le droit de nommer et de déplacer les ingénieurs. Ainsi commence à s'imposer en France l'idée que tous les 
élèves, sans aucune exception, reçoivent le même enseignement afin de former un corps d'ingénieurs homogène quant aux savoirs et qui puisse être envoyé partout en France pour établir le réseau routier suivant les mêmes techniques. Parallèlement, l'uniformisation du mode de financement se poursuit. Les Constituants remplacent les divers moyens de financement des travaux routiers en usage sous l'Ancien Régime (imposition, emprunt, péage, etc.) par le produit des sols additionnels perçus localement. S'il peut passer pour un exemple de décentralisation quant à la répartition des fonds comparé à l'Ancien Régime, ce système s'inscrit d'un autre côté dans une vision globale qui prône à l'inverse une centralisation plus forte dans laquelle le département n'est qu'une partie de la France entière. Ce régime fiscal se veut plus égalitaire, plus contrôlable, plus uniforme et mieux corrélé à la fiscalité directe comme au revenu local. Nonobstant, le souci des révolutionnaires de ne pas risquer l'interruption du service ralentit la marche vers la centralisation et l'uniformisation, tant au niveau réglementaire que dans la pratique. Ayant déjà éprouvé la vacance du pouvoir administratif local pendant les années 1789 et 1790, le pouvoir parisien, désireux de lui rendre rapidement de l'activité, laisse provisoirement aux départements le choix des ingénieurs et l'organisation locale se fait ainsi avec l'approbation de l'administration centrale des ponts et chaussées. Si l'administration locale des ponts et chaussées est officiellement absorbée au niveau national, le personnel technique en activité depuis l'Ancien Régime reste bien en place.

Même si l'administration locale des travaux publics est soumise à une hiérarchie au sommet de laquelle se trouvent le ministre de l'Intérieur et le Corps des Ponts et Chaussées, elle ne perd pas tous ses droits concernant les travaux routiers et conserve même un certain niveau d'autonomie, comme en atteste l'instruction du 17 avril 1791, qui définit pour la première fois le mode de fonctionnement de l'administration en la matière. Naturellement, cette autonomie n'a plus rien à voir avec celle des États de Languedoc. Elle se borne aux limites définies par l'administration centrale des ponts et chaussées et aux compétences que celle-ci abandonne aux départements. Comme sous l'Ancien Régime, tous les projets impliquant un choix des routes à construire ou à entretenir sont à la charge de l'administration locale et des ingénieurs qui y sont rattachés. Selon la hiérarchie locale, et après l'approbation du directoire du département qui prend l'arrêté en consultant l'avis de l'ingénieur en chef, le projet est renvoyé au directoire du district pour procéder à l'adjudication. Si elle juge nécessaire de solliciter l'assistance technique de l'Assemblée des Ponts et Chaussées, l'administration départementale transmet les projets à Paris. Cela témoigne du rôle de cette assemblée comme référence technique à l'échelle nationale, mais l'envoi des projets des travaux à Paris ne constitue pas une obligation administrative. Autrement dit, l'autorisation de l'administration centrale des ponts et chaussées ne constitue pas un préalable pour les travaux particuliers des routes. Pourtant, sur le plan budgétaire, il est vrai que l'influence de Paris est renforcée. La comptabilité annuelle des travaux publics, dressée par l'ingénieur en chef et approuvée par le directoire du département, doit être envoyée en janvier de chaque année au ministre de l'Intérieur. De même, l'administration locale doit informer le ministre sur le budget annuel des travaux publics du département, voté par le conseil général. Même si le directoire dispose institutionnellement d'un certain niveau d'autonomie, toutes les informations sur les travaux publics sont envoyées à Paris et, par ce moyen, le ministre de l'Intérieur peut intervenir dans l'établissement du budget des ponts et chaussées du département. Quoique le conseil général détienne le droit de voter définitivement le budget 
départemental, la surveillance de la part de l'administration centrale est bien plus forte que sous l'Ancien Régime.

6 Dans les faits, l'enjeu le plus important dans le renforcement du contrôle de l'administration centrale des ponts et chaussées au niveau du financement réside dans le degré d'initiative des différents acteurs des travaux routiers. Si l'administration du département de la Haute-Garonne maintient une certaine autonomie, ce n'est que dans la mesure où il parvient à se procurer les moyens financiers de sa politique ; or, c'est sur les reliquats du Languedoc et des commissions provisoires que doivent compter des administrateurs qui, par ailleurs, se plaignent toujours de l'insuffisance des fonds alloués aux travaux publics tant par le ministre de l'Intérieur que par le conseil général. Déjà sous l'Ancien Régime, l'existence de fonds laissés à la disposition de l'administration locale peut être considéré comme un bon thermomètre de son autonomie. Alors que la perception des nouvelles contributions directes ne cesse d'être retardée, les résidus fournissent au département le moyen de faire les travaux. Il est vrai que, sous l'impact de l'inflation, des demandes d'indemnité des entrepreneurs et de leur morosité, les dépenses tendent à baisser, laissant en souffrance une partie des fonds disponibles, mais, au moment de l'établissement du budget annuel, les résidus tant de l'Ancien Régime que des années précédentes rendent budgétairement envisageables de projeter des travaux pour une valeur supérieure au seul produit des sols additionnels de l'exercice en cours.

7 Dans le département de la Haute-Garonne, l'activité des travaux pendant la décennie révolutionnaire atteint son point culminant après la phase préparatoire de 1791-1792, quand tous les reliquats ont pu être accumulés. Cette activité tient non seulement à l'abondance des résidus, mais aussi, il faut le remarquer, aux résultats plus ou moins tangibles de la politique du département envers les entrepreneurs, politique dans laquelle l'État laisse au département les coudées franches. C'est en effet au pouvoir local de faire face à toutes les situations dans le cadre de la réglementation générale définie par le pouvoir parisien à cet égard. L'administration départementale, qui met l'accent sur la continuation des travaux, essaie d'apaiser les entrepreneurs en leur accordant une indemnité et en leur offrant de nouvelles conditions, pour mieux les sommer, d'autre part, par des mesures administratives et judiciaires, de continuer leurs chantiers. Néanmoins, les compétences du département dans le domaine des travaux publics sont limitées institutionnellement par les Constituants - qui contrôlent le volant de financement en assignant un montant maximum aux sols additionnels -, par l'administration centrale des ponts et chaussées - qui donne en pratique l'avantage aux travaux déjà commencés sur les ouvrages neufs -, et, enfin, par la règle générale qui s'impose aux départements de réduire la charge fiscale pesant sur la population par rapport à l'Ancien Régime et de la plafonner en fonction de « la force contributive » des habitants.

8 Pendant les trois ans que dure ce régime des sols additionnels au sein de la nouvelle administration départementale, le pouvoir central, malgré ses efforts de centralisation et d'uniformisation, n'est pas encore assez fort pour intervenir dans toutes les affaires de travaux routiers. L'État ne détient ni le droit d'établir définitivement les budgets départementaux, ni celui de répartir à son gré ces ressources entre tous les départements. En somme, si le pouvoir local maintient donc une certaine autonomie, ce n'est que dans la mesure où le manque de moyens du nouveau pouvoir parisien autorise la survivance des aspects de l'Ancien Régime favorables à cette marge d'autonomie. 
9 Le renforcement du contrôle de l'autorité centrale reçoit une impulsion nouvelle avec le décret du 16 frimaire an II, par lequel les Conventionnels se fixent l'objectif ambitieux de voir les routes et les ponts de la République entièrement réparés à ses frais. Le pouvoir législatif et l'administration centrale prennent désormais en mains la distribution des fonds pour les travaux publics et, par l'intermédiaire du suivi de ces fonds, ils s'insinuent dans le choix des travaux à exécuter. Leur surveillance s'étend même aux détails des dépenses réalisées. Pour toute réparation ou reprise de construction, l'autorisation de l'administration centrale devient un préalable et ce principe, établi à l'époque du légicentrisme et des commissions exécutives, perdure sous le régime ministériel restauré par la constitution de l'an III. Le désir du pouvoir central de contrôler tous les travaux publics routiers du pays et de concentrer les moyens limités dont il dispose sur ceux qui lui semblent les plus essentiels à l'intérêt général survit donc à l'expérience du gouvernement révolutionnaire. Par ailleurs, les traitements du personnel technique étant à la charge du Trésor public, il convient de les modifier en fonction de la hausse des prix et le pouvoir exécutif entend surtout les fixer pour les conducteurs en cohérence avec les salaires des autres fonctionnaires. Les conditions de travail des techniciens sont ainsi révisées et normalisées. De plus, à partir de la fin de l'an IV, le ministre de l'Intérieur assume de plus en plus la direction des carrières professionnelles des ingénieurs.

10 Avec ce degré sans précédent de centralisation, les compétences du directoire du département sont d'autant plus réduites qu'il ne dispose plus que des fonds qui lui sont versés par le Trésor public. Les résidus des années antérieures à 1793 ne sont désormais appliqués qu'à l'indemnité des entrepreneurs pour les ouvrages réalisés avant et pendant cette même année. L'administration locale ne conserve que l'initiative de proposer à Paris les travaux à faire et de prendre les mesures nécessaires pour presser les entrepreneurs afin d'accélérer l'exécution des travaux, alors même qu'il devient de plus en plus difficile de leur faire remplir les obligations de leurs baux. Malgré le contrôle renforcé de l'État sur les travaux routiers au niveau institutionnel, le défaut de fonds dans le département, résultant de l'inflation et de la défaillance financière de l'État, ne permet pas d'attacher les entrepreneurs aux chantiers, alors même que le renouvellement des baux est exclu.

11 Pour les autorités parisiennes, une centralisation renforcée est le meilleur moyen de faire valoir l'intérêt général de la République. À leurs yeux, si l'on laisse aux administrations départementales l'initiative en la matière, leurs mesures ne répondront nécessairement qu'à des intérêts locaux. L'idée selon laquelle seul le pouvoir parisien peut juger de l'intérêt général en matière routière et qu'il convient de concentrer tous les efforts sur les travaux essentiels se forme en 1792-1793, quand la guerre s'impose au dehors puis au dedans. À partir de ce moment, le critère militaire l'emporte sur celui du commerce en matière de politique routière. La priorité des travaux obéit aux itinéraires des convois militaires plutôt qu'à l'importance des lieux que les chemins desservent. La centralisation de l'administration des ponts et chaussées est justifiée et accélérée par la nécessité de concentrer les moyens sur l'entretien des routes traversant les départements frontières. De plus, d'un autre point de vue, c'est la centralisation qui rend possible cette concentration des fonds. Le contrôle des autorités parisiennes sur la répartition des fonds, ainsi que la priorité donnée aux routes d'intérêt stratégique, ont pour conséquence de négliger relativement les départements qui, comme la Haute-Garonne, ne sont pas sur le théâtre 
des opérations. En effet, à partir de 1794, le département toulousain ne reçoit qu'une somme relativement modique pour les travaux routiers par rapport aux départements de nord-est de la France, notamment celui du Nord.

Par conséquent, quant aux travaux des ponts et chaussées, le directoire du département devient un intermédiaire actif, c'est-à-dire conservant un rôle politique limité mais important dans l'initiative des travaux, mais dominé par un pouvoir central qui le borne à exécuter sous son contrôle des travaux conformes à l'intérêt supérieur de la République. Insérée dans un nouvel ordre territorial uniforme destiné à liquider les particularités provinciales de l'Ancien Régime, l'administration départementale, dans le contexte politique et militaire de la Révolution française, ne peut que se plier à la politique routière imposée par l'administration centrale. En ce sens, ce sont les circonstances, notamment la guerre révolutionnaire, qui imposent, du point de vue des travaux publics, le projet de perte d'autonomie et l'intégration à la France des départements porté par les Constituants. Cette tendance ne permet pas au département de relever rapidement et efficacement les défis de l'inflation, de la pénurie des ouvriers et de la morosité des entrepreneurs.

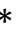

13 L'époque révolutionnaire est donc une période durant laquelle la centralisation et l'uniformisation sont incontestablement accélérées. Ce constat nous invite à repenser les notions de départementalisation ou de décentralisation que Jean Pétot et JeanMarcel Goger mobilisent pour décrire la situation de l'administration des ponts et chaussées en 1791. Pour eux, l'État laisse la responsabilité de l'entretien et du financement des routes à chaque département. Pourtant, il n'est pas certain que l'organisation des ponts et chaussées du début de la Révolution française soit moins centralisée de ce point de vue que celle de l'Ancien Régime. Même si l'administration des ponts et chaussées mêle les deux tendances, centralisatrice et décentralisatrice, dans ses différents aspects, la Révolution française nous semble plutôt correspondre à un progrès de la centralisation. Malgré l'instabilité financière, préoccupation majeure des administrateurs tant locaux que nationaux, ces derniers agissent dans le sens d'une uniformisation du système des travaux publics à l'échelle nationale qui, contrairement au processus de centralisation à l'œuvre sous l'Ancien Régime, qui s'accommode des pays d'états, ne souffre plus aucune exception. Du point de vue des travaux publics, la départementalisation se borne à un changement de cadre administratif.

14 Là où Michel Biard, dans ses travaux sur les représentants du peuple en mission, conclut à la nécessité de remettre en cause le concept de "centralisation jacobine », le cas des ponts et chaussées montre comment la centralisation peut s'opérer sans ingérence d'un agent de l'État dans les décisions des administrateurs locaux ou pour forcer l'exécution des lois, mais de manière indirecte et implicite : dans ce domaine technique, qui suppose des savoirs que les administrateurs ne maîtrisent généralement pas, ces derniers dépendent de l'expertise d'un personnel technique, les ingénieurs, sur lequel l'administration centrale renforce sa direction. Bien sûr, il ne serait pas correct de qualifier cette centralisation de l'administration des ponts et chaussées de l'an II de «jacobine », expression péjorative destinée davantage à stigmatiser la période dite de la Terreur qu'à la décrire, dans la mesure où cette centralisation, s'il est indéniable 
qu'elle s'accélère en l'an II, commence dès le début de la Révolution française. En outre, elle survit durablement à la réaction thermidorienne et à la suppression du système des commissions exécutives, assimilé par ses détracteurs à une administration pléthorique et à une économie dirigée qui n'est alors plus de mode. C'est sans doute le contexte militaire qui explique le maintien de cette centralisation des ponts et chaussées : l'attention particulière portée aux routes empruntées par les convois militaires est une constante de la politique gouvernementale, aussi bien à l'époque du comité de Salut public que du Directoire.

\section{AUTEUR}

\section{DAEBO KIM}

Docteur en histoire

IHRF-IHMC

Université Paris 1 Panthéon-Sorbonne 